\section{Prevalencia de la obesidad en el Uruguay}

La población del Uruguay asciende a poco más de 3 millones de habitantes. Es predominantemente de raza blanca, ya que la mayor parte desciende de inmigrantes españoles, italianos y centroeuropeos. Pocos uruguayos son de descendencia indígena o africana. La mayoría pertenecen a la clase media y $40 \%$ viven en Montevideo, la capital. Cerca de $50 \%$ de los habitantes buscan atención médica en los hospitales públicos, $40 \%$ en las organizaciones de seguros médicos y $10 \%$ acuden a médicos particulares. Como ocurre hoy en día en muchas partes del mundo, los problemas asociados con la obesidad han cobrado mucha importancia en el Uruguay. A continuación se resumen datos obtenidos de investigaciones epidemiológicas llevadas a cabo a fines del siglo XX y se comparan con otros de 10 años antes. En las investigaciones más recientes se emplearon las definiciones de "sobrepeso" y "obesidad" establecidas por el Grupo de Trabajo sobre Obesidad de la OMS: sobrepreso, índice de masa corporal (IMC) de 25 a $29,9 \mathrm{~kg}$ por metro cuadrado $\left(\mathrm{m}^{2}\right)$ de superficia corporal; obesidad, IMC de $30+\mathrm{kg} / \mathrm{m}^{2}$.

En 1999, las prevalencias de sobrepeso y obesidad en los adultos fueron de $34 \%$ y $17 \%$, respectivamente, y el total de ambas, de $51 \%$. Estas cifras se obtuvieron gracias a la encuesta transversal llevada a cabo en una muestra sistemática, aleatoria y estratificada de 900 personas (392 varones y 508 mujeres) mayores de 18 años que residían en la capital y en ciudades de más de 10000 habitantes. Miembros de un equipo adiestrado de encuestadores realizaron las entrevistas en los hogares de los participantes.

En el año 2000, las prevalencias de sobrepeso y obesidad en los niños ascendían a $17 \%$ y $9 \%$, respectivamente, o a $26 \%$ en total. Estas cifras se consiguieron mediante una encuesta transversal de 886 niños (452 varones y 434 niñas) de 9 a 12 años de edad residentes en la capital y otras ciudades de más de 10000 habitantes. Se visitó a los niños en los hogares, en presencia de uno de los padres. Los niños fueron pesados, medidos y entrevistados por miembros de un equipo de investigadores adiestrados para ese fin.

A diferencia de las definiciones de sobrepeso y obesidad empleadas en 1999 y 2000, la de 10 años antes fue diferente. En 1989 se hizo una encuesta de adultos (2 114 mujeres y 2249 hombres) que habitaban en Montevideo. Los participantes se seleccionaron para la encuesta cuando acudieron al cuartel central de la policía para adquirir carnés de identidad. El estudio de tres meses estuvo a cargo de estudiantes universitarios de la Escuela de Nutrición y Dieta. Se encontró que $49,8 \%$ de las mujeres y $41,8 \%$ de los varones tenían más de $10 \%$ de peso excesivo comparados con la escala del peso ideal usada por el seguro metropolitano. Si bien la fórmula para peso excesivo de 1989 no es totalmente comparable con la de los estudios de 1999 y 2000, sirve para poner en claro que había una alta prevalencia de obesidad.

Por las cifras presentadas, puede concluirse que el Uruguay tiene una elevada incidencia de sobrepeso y obesidad tanto en adultos como en niños. Más de la mitad de la población adulta y más de una cuarta parte de los niños adolecían de peso excesivo al comenzar el siglo XXI. A pesar de los esfuerzos invertidos en educar a los escolares sobre las desventajas de la gordura, tal parece que no hubo ningún cambio importante en la prevalencia del peso excesivo en los 10 años que transcurrieron entre encuestas. (Núñez Rocha GM et al. Prevalence of obesity in Uruguay. Obesity. 2004;5: 175-176.)

\section{Análisis constructivista sobre el éxito de la maternidad sin riesgo en Honduras}

Se estima que anualmente mueren por complicaciones del parto de 500000 a 600000 mujeres. Se trata de una de las causas principales de muerte durante la etapa reproductiva, es decir, en plena juventud. En 1987 se lanzó una iniciativa mundial para combatir el problema pero, a partir de esa fecha, pocos países en desarrollo registraron una reducción importante de muertes maternas. Honduras es la excepción. Entre 1990 y 1997, el índice de mortalidad materna en ese país, o sea el número de muertes por complicaciones durante el embarazo, parto y puerperio por cada 100000 nacidos vivos, disminuyó de 182 a 108 (40\%). Esta es una de las reducciones más grandes jamás documentadas en tan poco tiempo en un país en desarrollo. El motivo 